Int. J. Morphol.,

33(3):914-919, 2015.

\title{
Características Biométricas de los Osículos del Oído de Individuos Chilenos
}

\author{
Biometric Characteristics of Ear Ossicles in Chilean Subjects
}

Binvignat, O.*; Meza, S..*; Torrez, J. C.*** \& Olave, E.***

BINVIGNAT, O.; MEZA, S.;TORREZ, J. C. \& OLAVE, E. Características biométricas de los osículos del oído de individuos chilenos. Int. J. Morphol., 33(3):914-919, 2015.

RESUMEN: El crecimiento y desarrollo de los osículos del oído medio no muestran grandes variaciones después del nacimiento. Nuevas tecnologías en cirugía de implantes, corrección y tratamiento de las enfermedades del oído medio necesitan un detallado conocimiento biométrico de estos osículos. Basados en lo anterior, se estudiaron 22 Malleus, 16 Incus y 3 Estapedios de individuos adultos de sexo masculino, de cadáveres secos, todos individuos de la Región Central de Chile. Los osículos fueron analizados y fotografiados con un Microscopio quirúrgico de diagnóstico OPMI Pico, Carl Zeiss, imagen estereoscópica. Los datos métricos se obtuvieron con un calyper digital Mitutoyo y sometidos a análisis digital con el AxioVision LE 4.8.2.0. Software de Carl Zeiss. El malleus tuvo una longitud promedio desde la cabeza al ápice del manubrio de $8,08 \mathrm{~mm}$ y su proceso anterior una longitud de $0,76 \mathrm{~mm}$; el proceso largo del incus tuvo una longitud promedio de $3,37 \mathrm{~mm}$ y el proceso corto de $3,07 \mathrm{~mm}$; la base del estapedio tuvo una longitud antero-posterior de 2,53 mm. Los resultados obtenidos son un aporte al conocimiento de los osículos del oído en nuestra población.

PALABRAS CLAVE: Anatomía; Biometría; Oído medio; Osículos.

\section{INTRODUCCIÓN}

El oído medio ha sido objeto de observación y estudio por diversos anatomistas. El estudio de estos osículos datan del Siglo XVI, cuando Vesalius describió el incus y el malleus en 1543 (Cole, 1949) e Ingrassias y Eustachius describieron el estapedio en 1546 y 1564, respectivamente, según ha sido mencionado en el trabajo de Harneja \& Chaturvedi (1973).

La mayoría de estos investigadores de la edad media y posteriormente anatomistas modernos, reportaron la forma y una serie de registros métricos de los osículos del oído medio y aunque recientemente las informaciones han aumentado, aún es necesario complementar con otros parámetros relacionados a su biometría. La morfología y biometría de los osículos del oído medio en humanos es de interés, tanto desde el punto de vista quirúrgico como también para el diseño y reconstrucción de implantes electromagnéticos, que debe usar medidas precisas para lograr la reproducción y la transmisión de la energía sonora (Mills, 1991; Nishihara \& Goode, 1994; Asai et al., 1999; Whyte et al., 2008; Murugasu et al., 2005; Farahani \& Nooranipour, 2008).
Su crecimiento y desarrollo también han sido objeto de diversos estudios, describiéndose que el malleus, incus y parcialmente el estapedio tienen osificación endocondral (Whyte et al.) y algunos de los detalles óseos, especialmente el proceso anterior del malleus tiene una osificación intramembranosa proveniente de células mesenquimatosas (Rodríguez-Vásquez et al., 1993; Whyte et al.; Ramírez \& Ballesteros, 2013a, 2013b).

Basados en lo anterior y con el propósito de complementar el conocimiento biométrico de estos osículos en la población Chilena, estudiamos sus características y registramos diversos parámetros de ellos, que esperamos sean un aporte para los profesionales que se desenvuelven en el área otológica.

\section{MATERIAL Y MÉTODO}

En el presente estudio se utilizaron cráneos retirados de osamentas humanas que estaban siendo removidas de nichos temporales del Cementerio Municipal de la ciudad de

\footnotetext{
* Profesor Participante en Programa de Magister en Ciencias, mención Morfología, Universidad de La Frontera, Chile.

** Universidad Autónoma de Chile, Sede Talca, Chile.

${ }^{* * *}$ Facultad de Medicina, Universidad de La Frontera, Temuco, Chile.
} 
Talca-Chile. Estos correspondían a individuos de sexo masculino de edades comprendidas entre los 40 y 70 años (análisis post mortem). Manualmente, los osículos fueron retirados del oído medio y se limpiaron parcialmente con aire y un pincel, retirando restos de tierra o polvo.

Se obtuvieron 22 Malleus (12 Derechos y 10 izquierdos), 16 Incus ( 8 derechos y 8 izquierdos) y 3 Estapedios (2 derechos y 1 izquierdo).

Los osículos fueron identificados y clasificados según lateralidad, luego micro fotografiados con un microscopio quirúrgico de diagnóstico OPMI Pico, Carl Zeiss, a un aumento $1.6 \mathrm{x} \mathrm{y}$, osteo-métricamente analizados con un paquímetro electrónico marca Mitutoyo. También fueron sometidos a un análisis digital con el software AxioVision L.E 4.8.2.0. Software de Carl Zeiss.

Diversas medidas fueron obtenidas de la distancia entre puntos osteométricos de los osículos. Las mediciones registradas fueron las siguientes:

\section{Malleus}

a.- Longitud de la cabeza

b.- Ancho de la parte media de la cara lateral

c.- Ancho anteroposterior de la cabeza

d.- Longitud de la zona articular de la cabeza

d.1. Ancho de la parte superior de la zona articular

d.2. Ancho de la parte media de la zona articular

d.3. Ancho de la parte inferior de la zona articular

e.- Ancho del cuello a nivel de la parte media de su cara lateral

f.- Longitud del proceso anterior

g.- Distancia entre los ápices del manubrio y del proceso anterior

h.- Longitud del manubrio desde su ápice hasta el cuello del osículo

i.- Distancia entre el ápice del manubrio y extremo de la cabeza (longitud del osículo)

j.- Ancho del manubrio a nivel de la parte inferior de la base del proceso anterior

k.- Ancho del manubrio a nivel de su parte media

1.- Ancho del manubrio, próximo a su ápice

\section{Incus}

a.- Longitud del proceso largo

b.- Longitud del proceso corto

c.- Ancho a nivel de la base de los procesos largo y corto

d.- Ancho, próximo al ápice de estos procesos

e.- Distancia entre ápice del proceso corto a la carilla incudomaleolar (por su cara medial)

e.1. Ápice-margen superior de la carilla

e.2. Ápice-parte media de la carilla e.3. Ápice-margen inferior de la carilla

f.- Distancia entre ápice del proceso largo a la carilla incudomaleolar (por su cara medial)

f.1. Ápice-margen superior de la carilla

f.2. Ápice-parte media de la carilla

f.3. Ápice-margen inferior de la carilla

g.- Ángulo a nivel de la unión de los procesos largo y corto

\section{Estapedio}

a.- Longitud anteroposterior de la base

b.- Ancho en su parte media

c.- Ancho en su parte anterior

d.- Ancho en su parte posterior

e.- Distancia entre las partes internas de los ramos anterior y posterior

f.- Distancia entre la parte central del arco formado por sus dos ramos y su base

g.- Distancia entre la parte articular de la cabeza y su base

h.- Ancho de los ramos anterior y posterior

Los datos obtenidos fueron digitados en el programa Excel y sometidos a un análisis estadístico simple, para obtener promedios y desviación estándar. También con el software Prisma 6.0 (Graph Pad Software, San Diego CA.) un análisis de significación para comparación con otros datos obtenidos de la literatura.

\section{RESULTADOS}

Con respecto a los registros métricos del malleus (Fig. 1a), la longitud de su cabeza fue en promedio de 3,33 mm (DE 0,37); el ancho en la parte media de su cara lateral fue de 2,37 mm (DE 0,15); el ancho antero-posterior de la cabeza fue de 1,63 mm (DE 0,2); la zona articular de la cabeza tuvo una longitud promedio de 2,80 $\mathrm{mm}$ (DE 0,28). El ancho del malleus a nivel de la parte superior de esta zona articular tuvo en promedio 1,31 mm (DE 0,19); a nivel de la parte media fue de $1,63 \mathrm{~mm}(\mathrm{DE} 0,2)$ y a nivel de la parte inferior fue de $0,82 \mathrm{~mm}(\mathrm{DE} 0,23)$. El cuello del malleus a nivel de la parte media de su cara lateral fue de $0,95 \mathrm{~mm}$ (DE 0,09$)$. El proceso anterior tuvo una longitud promedio de $0,76 \mathrm{~mm}(\mathrm{DE} 0,16)$.

La distancia entre el ápice del manubrio y el ápice del proceso anterior fue de 4,58 $\mathrm{mm}$ (DE 0,43); la longitud promedio del manubrio considerando este parámetro desde su ápice hasta el cuello fue de 4,26 mm (DE 0,33); la distancia entre el ápice del manubrio y el extremo de la cabeza del malleus (longitud del osículo) fue de 8,08 (DE 0,28). La proporción que obtuvimos entre longitud del manubrio y la longitud total del osículo fue de 52,7\%. 


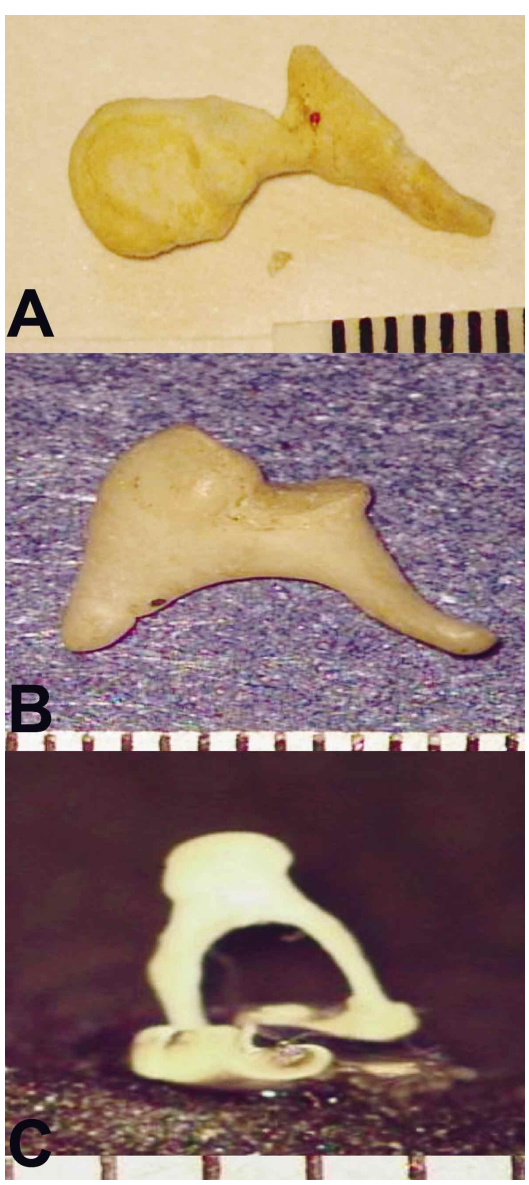

Fig. 1. Osículos del oído. a). Malleus, b). Incus y c). Estapedio.

El ancho del manubrio a nivel de la parte inferior de la base del proceso anterior fue de 1,21 mm (DE 0,17), a nivel de de su parte media de $1,19 \mathrm{~mm}$ $(\mathrm{DE} 0,2)$ y de la parte próxima al ápice de $0,58 \mathrm{~mm}(\mathrm{DE} 0,07)$.

En relación a los valores obtenidos de las mediciones del incus (Fig. 1b), la longitud promedio de su proceso largo fue de $3,37 \mathrm{~mm}(\mathrm{DE} 0,42)$ y de su proceso corto fue de 3,07 mm (DE 0,33). La distancia entre los ápices de los procesos largo y corto fue de $5,45 \mathrm{~mm}$ (DE 0,49).

El ancho promedio próximo al ápice de los procesos largo y corto fueron $0,62 \mathrm{~mm}(\mathrm{DE} 0,1)$ y $0,97 \mathrm{~mm}(\mathrm{DE}$ $0,16)$, respectivamente; a nivel de la base fueron $0,96 \mathrm{~mm}(\mathrm{DE} 0,15)$ y 1,6 $\mathrm{mm}(\mathrm{DE} 0,21)$, respectivamente.
Las distancias promedios desde el ápice del proceso corto a la carilla articular incudo-maleolar, registros hechos por su cara medial, fueron las siguientes: ápice-margen superior de la carilla 5,05 mm (DE 0,35), ápice-margen de zona media de la carilla 4,11 mm (DE 0,26) y ápice-margen inferior de la carilla 5,27 mm (DE 0,23).

Las distancias promedios desde el ápice del proceso largo a la carilla incudomaleolar, también medidos por su cara medial, fueron las siguientes: ápice-margen superior de la carilla 6,21 mm (DE 0,53), ápice-margen de la parte media de la carilla 4,11 mm (DE 0,62) y ápice-margen inferior de la carilla 3,89 $\mathrm{mm}(\mathrm{DE} 0,45)$.

El ángulo promedio que forman la unión de los procesos largo y corto fue de 119,5 grados (DE 10,78) y el ángulo que forman las dos partes de la carilla articular fue de 100,04 grados (DE 12,28).

Con respecto al osículo estapedio (Fig. 1c), tiene una cabeza, cuello, ramo anterior y posterior y una base. La base tiene una longitud promedio en sentido antero-posterior de $2,53 \mathrm{~mm}(\mathrm{DE} 0,14) \mathrm{y}$ un ancho promedio en su parte media de $1,21 \mathrm{~mm}$ (DE 0,03); un ancho en la parte media de su zona anterior de 1,23 mm (DE 0,09$)$ y un ancho en la parte media de su zona posterior de 1,09 mm (DE $0,02)$.

La distancia entre las partes internas de los ramos anterior y posterior, por sobre la base, fue de 1,54 mm (DE 0,05); la distancia entre la parte central del arco formado por la unión de los dos ramos y su base fue de $1,73 \mathrm{~mm}(\mathrm{DE} 0,07)$ y la distancia entre la parte articular de su cabeza y su base fue de 3,01 mm (DE $0,03)$.

El grosor antero-posterior del ramo anterior fue de $0,42 \mathrm{~mm}(\mathrm{DE} 0,03)$ y el del ramo posterior fue de $0,59 \mathrm{~mm}$ (DE 0,04).

\section{DISCUSIÓN}

Los osículos del oído medio han sido motivo de estudio tanto en su morfología como en su biometría en diversos períodos de la vida y se ha evidenciado una leve variación de sus medidas después del nacimiento (Olsewski, 1990).

Las técnicas quirúrgicas necesitan de datos morfométricos más acuciosos que posibiliten la reconstrucción y diseño iconográfico de implantes (Whyte et al.; Murugasu et al.; Farahani \& Nooranipour).

Respecto al malleus, entre los parámetros registrados en nuestra serie, la longitud entre cabeza y ápice del manubrio (longitud total) tuvo un promedio de $8,08 \mathrm{~mm}$ y la distancia entre este último y el proceso lateral (longitud del manubrio) fue de 4,26 mm, cuyas medidas son comparables con otros estudios: 7,9 y 4,7 $\mathrm{mm}$ respectivamente (Bouchet \& Giraud, 1968); 8,0 y 4,2 mm (Harada \& Ishii, 1972); 7,3 y 3,5 mm (Arensburg et al.,1981); 8,1 y 4,9 mm (Aycan et al., 1990); 7,7 y 4,7 mm (Unur et al., 2002); 7,86 y 4,46 mm (Crevecoeur, 2007); 8,25 y 4,94 mm (Quam \& Rak, 2008); 8,1 y 4,91 mm (Flohr et al., 2010); 7,95 y 4,76 mm (Singh et al., 2012), y 8,53 y 5,2 mm (Mogra et al., 2014). 
Así como éstos últimos autores que estudiaron a un grupo de individuos de la India, Vinayachandra et al. (2014) en otro grupo, registraron para la longitud del malleus un promedio un poco menor de $7,37 \mathrm{~mm}$ en el lado derecho y de 7,51 en el izquierdo.

La longitud total de este osículo fue calculada a través de fotografías por Quam \& Rak, quienes obtuvieron valores entre 7,43-9,31 $\mathrm{mm}$, pero su promedio de 8,25 $\mathrm{mm}(\mathrm{DE}$ 0,41) fue similar al nuestro. Valores menores han sido informados también, por mencionar a algunos, Harneja \& Chaturvedi informaron 7,15 mm (DE 0,3 ) de promedio y Vinayachandra et al. registraron para la longitud del malleus un promedio de 7,37 $\mathrm{mm}$ en el lado derecho y de 7,51 $\mathrm{mm}$ en el izquierdo.

El promedio obtenido en nuestra investigación es muy cercano al de Flohr et al. quienes tuvieron 8,11 mm (DE $0,37)$ para este parámetro.

Como se puede apreciar, los diferentes valores informados para la longitud de este osículo difieren levemente unos de otros, encontrándose una diferencia en lo informado por Mogra et al. y Vinayachandra et al., considerando que ambos estudios fueron realizados en población de la India.

Este hueso ha sido estudiado con propósitos de utilizarlo como herramienta para determinar el sexo de individuos con el cuerpo severamente mutilado o descompuesto, encontrando que sí podría llevar a una conclusión determinante, considerando la longitud del hueso y la longitud del manubrio (Singh et al.). Sin embargo, dejan en claro que sus hallazgos deberían ser replicados en otras poblaciones y en diferentes partes del mundo. Por otra parte, Ishimoto et al. (2004) han establecido que el canal auditivo externo juega un rol importante en el desarrollo y localización del manubrio maleolar.

Para la longitud entre la cabeza y el nivel de la raíz del proceso anterior (longitud del manubrio) hubo igualmente similitudes con Quam \& Rak quienes determinaron rangos de 4,22-5,59 mm, donde su promedio fue de 4,94 $\mathrm{mm}$ (DE $0,31)$. Harneja \& Chaturvedi mostraron medidas cercanas inferiores para el largo total de 4,22 mm (DE 0,35). Como variación se podría contar con el registro de la medida más pequeña de esta distancia, $3,5 \mathrm{~mm}$.

El estudio de Crevecoeur incluyó también el ancho medio-lateral de la cabeza y el ancho antero-posterior de la cabeza en una muestra que fue de 2,6 y $2,1 \mathrm{~mm}$, respectivamente, resultado diferente al promedio de nuestro estudio, para las mismas medidas, de $2,37 \mathrm{~mm}$ (DE 0,15$)$ y $1,63 \mathrm{~mm}$
(DE 0,2), respectivamente. Quam \& Rak obtuvieron para el ancho medio-lateral un promedio de 2,43 mm (DE 0,17), valor concordante con el de nuestra serie.

Quam \& Rak, midieron también el ancho medio-lateral del cuello (registro latero-lateral) con un valor promedio de $0,99 \mathrm{~mm}(\mathrm{DE} 0,13)$ y en nuestra serie se obtuvo un valor de $0,95 \mathrm{~mm}$ (DE 0,09). En cuanto a variaciones de este hueso, no encontramos ninguna característica significativa, confirmando lo expresado por Sarrat et al. (1992) y Unur et al., que el malleus tiene una morfología estable, incluyendo los cambios postnatales (Olsewski, 1990). Por su parte, Arensburg et al., también encontraron los osículos con morfometría muy estable en poblaciones con contrastes étnicos y de localización geográfica amplia.

Unur et al., describieron variaciones de longitud del proceso anterior en osículos de recién nacidos, muestras que no tuvimos en nuestra serie, donde nuestro resultado promedio fue de 0,76 mm (DE 0,16) en adultos. Ramírez \& Ballesteros (2013a) informaron para este parámetro en población Colombiana un promedio de 0,59 mm (DE 0,21).

Con respecto a las medidas obtenidas del incus en el presente estudio, algunas medidas pueden ser comparadas con estudios previos que corresponden en la mayoría a la longitud total del incus, o sea, desde el ápice del proceso largo al margen superior de la carilla articular y el ancho total, medido desde el proceso corto a la parte media del margen de la carilla, cuyos valores son 6,5 y $5,1 \mathrm{~mm}$, respectivamente, para Bouchet \& Giraud; 6,4 y $5,1 \mathrm{~mm}$ para Arensburg et al.; 6,5 y 4,9 mm para Unur et al.; 6,8 y 5,07 mm para Quam \& Rak; 6,7 y 5,2 mm para Flohr et al., y, 6,51 y $5,35 \mathrm{~mm}$ para Ramírez \& Ballesteros (2013b). El valor obtenido en nuestra serie fue de $6,21 \mathrm{~mm}$ y $5,05 \mathrm{~mm}$, respectivamente.

La distancia entre los ápices de sus procesos corto y largo fue en promedio de 4,2 $\mathrm{mm}$ (Harada), 6,1 $\mathrm{mm}$ (Unur et al.) y de 6,06 mm (Ramírez \& Ballesteros, 2013b), mientras que el obtenido en nuestra muestra fue de $5,45 \mathrm{~mm}$.

Para el ancho de la parte proximal (en la base de los procesos) y distal (próximo al ápice) de los procesos largo y corto, Ramírez \& Ballesteros (2013b) registraron para el proceso corto un promedio de 1,99 y 1,22 $\mathrm{mm}$, respectivamente; para el proceso largo su resultado fue de 1,25 y $0,78 \mathrm{~mm}$, respectivamente. Estos resultados difieren levemente de los obtenidos en nuestro estudio, ya que el ancho promedio próximo al ápice de los procesos corto y largo, fue de 0,97 y $0,62 \mathrm{~mm}$, respectivamente y el de la parte proximal fue de 1,6 y 0,96 $\mathrm{mm}$, respectivamente. 
El estudio de Flohr et al., tiene muchos parámetros informados, teniendo entre ellos, las medidas de longitud desde el ápice del proceso corto a la cresta anterior de la carilla articular de la articulación incudo-maleolar y la longitud desde el ápice del proceso corto a la carilla incudo-maleolar media caudal. Para la primera el promedio obtenido por estos autores fue de $5,19 \mathrm{~mm}$ y para la segunda, de 4,59 mm. Nuestros resultados fueron para la primera de las medidas $5,05 \mathrm{~mm}$ y para la segunda, 5,27 $\mathrm{mm}$.

Las distancias desde el ápice del proceso largo al margen de la parte media de la carilla incudomaleolar anterior, también medidos por su cara medial fue de 4,11 $\mathrm{mm}(\mathrm{DE} 0,62)$ y ápice-margen inferior de la carilla 3,89 mm (DE 0,45). Para la primera medida, Ramírez \& Ballesteros (2013b) obtuvieron un valor de 3,92 mm. Para la segunda medida, Quam \& Rak obtuvieron un promedio de 4,01 mm, Florh et al., 3,87 mm y Ramírez \& Balleste$\operatorname{ros}(2013 \mathrm{~b}), 4,66 \mathrm{~mm}$.

En general el incus es un osículo, que al compararlo con estudios realizados en otras poblaciones, no presenta grandes variaciones tanto en su morfología como en los parámetros biométricos

En relación al estapedio es el osículo que presenta mayores variaciones dentro de los osículos del oído medio, ya sea de su cuello, sus ramos (símétrico o asimétrico) o de su foramen (Unur et al.). Estos autores registraron un promedio de $3,22 \mathrm{~mm}$ para su altura, una longitud de su base de 2,57 $\mathrm{mm}$ y el ancho de su base de $1,29 \mathrm{~mm}$. Por su parte, Arensburgh et al. obtuvieron 3,2-2,6 y 1,8 $\mathrm{mm}$, respectivamente, valores con los cuales concordamos $(3,01-2,53-1,21 \mathrm{~mm}$, respectivamente)

Ramírez \& Ballesteros (2012) obtuvieron un promedio de $3,18 \mathrm{~mm}$ para la altura del estapedio, con 2,87 $\mathrm{mm}$ de longitud en su base y $1,15 \mathrm{~mm}$ de ancho, todos valores semejantes a Unur et al., y con los cuales también coincidimos.

Por otra parte, Farahani \& Nooranipour realizaron la medición del ángulo intercrural en 12 estapedios, utilizando microscopía electrónica y obtuvieron un ángulo promedio de 19,5 grados (DE 2,3). Revisando la literatura no se encontró información sobre esta variable y no fue calculado en nuestra serie.

Crevecoeur ha señalado que no existe correlación entre las dimensiones de los osículos con el tamaño del cráneo y el cuerpo. Arensburg et al., han indicado que los osículos tienen una morfometría muy estable en diversas poblaciones.
Un estudio de Olsewski (1989) mostró las variaciones de tamaño de partes de los osículos de niños hasta un año comparados con individuos adultos de edades entre 20 y 50 años, encontrando que hubo incremento de la longitud del malleus y del manubrio de éste en alrededor de un $12 \%$; de la longitud y ancho del incus en alrededor de $8 \%$ y de la longitud del estapedio y longitud de su base en alrededor de $5 \%$.

Finalmente, al hacer un análisis matricial sobre las mediciones y comparación en la lateralidad de los osículos del oído medio, no se encontró diferencia estadísticamente significativa.

En conclusión, encontramos que la morfología y morfometría del malleus e incus es homogénea y estable. Con respecto al estapedio, presenta mayores variaciones que los otros dos osículos, como ya fuera expresado por Unur et al.

El registro de todas las medidas óseas de los osículos del oído medio buscó corroborar y aportar a lo encontrado en otras investigaciones. De esta forma se amplía la información desde una perspectiva étnica y geográfica diferente, ya que no existen trabajos similares en la población Chilena.

BINVIGNAT, O.; MEZA, S.; TORREZ, J. C. \& OLAVE, E. Biometric characteristics of ear ossicles in Chilean subjects. Int. J. Morphol., 33(3):914-919, 2015.

SUMMARY: The growth and development of the ossicles of the middle ear do not show large variations after birth. New technologies in implant surgery, correction and treatment of diseases of the middle ear require an increasingly larger biometric knowledge of these ossicles. Therefore, we studied 22 Malleus, 16 Incus and 3 Stapes of male adult subjects, from dry corpses, and, one Malleus, one Incus and one Stapes of a seven-month-old male fetus, all Chilean subjects from the Central Region in Chile. These bones were analyzed and photographed with a model OPMI Microscope tilting binocular tube with stereoscopic 3D image. Metric data were obtained with the AxioVision L.E 4.8.2.0. Carl Zeiss software. The malleus had an average length from head to apex of $8.08 \mathrm{~mm}$, the anterior process had a length of $0.76 \mathrm{~mm}$, the long limb of the incus was an average length of $3.37 \mathrm{~mm}$ and the short limb $3.07 \mathrm{~mm}$, the base of the stapes measured $2.53 \mathrm{~mm}$. The results are a contribution to the knowledge of ear ossicles in our population.

KEY WORDS: Anatomy; Biometric parameters; Middle Ear; Ossicles. 


\section{REFERENCIAS BIBLIOGRÁFICAS}

Arensburg, B.; Harell, M. \& Nathan, H. The human middle ear ossicles: Morphometry, and taxonomic implications. J. Hum. Evol., 10(2):199-205, 1981.

Asai, M.; Huber, A. M. \& Goode, R. L. Analysis of the best site on the stapes footplate for ossicular chain reconstruction. Acta Otolaryngol., 119(3):356-61, 1999.

Aycan, K.; Unur, E. \& Bozkir, M. G. Anatomical study of malleus. J. Health Sci., 1:152-8, 1990.

Bouchet, A. \& Giraud, M. Contribution a l'etude morphologique et radiologique des osselets de l'ouie. Compte Rendu de l'Association des Anatomists 53 Congrés., 141:588-600, 1968.

Cole, F. G. History of Comparative Anatomy. London, McMillan \& Co. Ltd., 1949. pp.69-120.

Crevecoeur, I. New discovery of an Upper Paleolithic auditory ossicle: the right malleus of Nazlet Khater 2. J. Hum. Evol., 52(3):341-5, 2007.

Farahani, R. M. \& Nooranipour, M. Anatomy and anthropometry of human stapes. Am. J. Otolaryngol., 29(1):42-7, 2008.

Flohr, S.; Leckelt, J.; Kierdorf, U. \& Kierdorf, H. How reproducibly can human ear ossicles be measured? A study of inter-observer error. Anat. Rec. (Hoboken), 293(12):2094-106, 2010.

Harada, O. \& Ishii, H. The condition of the auditory ossicles in microtia: findings in 57 middle ear operations. Plast. Reconstr. Surg., 50(1):48-53, 1972.

Harneja, N. K \& Chaturvedi, R. P. A study of the human ear ossicles. Indian J. Otolaryngol. Head Neck Surg., 25(3):154-60, 1973.

Ishimoto, S.; Ito, K.; Kondo, K.; Yamasoba, T. \& Kaga, K. The role of the external auditory canal in the development of the malleal manubrium in humans. Arch. Otolaryngol. Head Neck Surg., 130(8):913-6, 2004.

Mills, R. P. Ossicular geometry and the choice of technique for ossiculoplasty. Clin. Otolaryngol. Allied Sci., 16(5):476-9, 1991.

Mogra, K.; Gupta, S.; Chauhan, S.;Panwar, L \& Rajuram. Morphological and morphometrical variations of malleus in human cadavers. Int. J. Healthc. Biomed. Res., 2(3):186-92, 2014.

Murugasu, E., Puria, S. \& Roberson, J. B. Jr. Malleus-to-footplate versus malleus-to-stapes-head ossicular reconstruction prostheses: temporal bone pressure gain measurements and clinical audiological data. Otol. Neurotol., 26(4):572-82, 2005.

Nishihara, S. \& Goode, R. L. Experimental study of the acoustic properties of incus replacement prostheses in a human temporal bone model. Am. J. Otol., 15(4):485-94, 1994.
Olsewski, J. Structure of the middle ear in infants. Otolaryngol. Pol., 43(4):278-83, 1989.

Olsewski, J. The morphometry of the ear ossicles in humans during development. Anat. Anz., 171(3):187-91, 1990.

Quam, R. \& Rak, Y. Auditory ossicles from southwest Asian Mousterian sites. J. Hum. Evol., 54(3):414-33, 2008.

Ramírez, L. M. \& Ballesteros, L. E. Human's stape anthropometry: A direct anatomic study. Int. J. Morphol., 30(4):1295-300, 2012.

Ramírez, L. M. \& Ballesteros, L. E. Anthropometry of the malleus in humans: A direct anatomic study. Int. J. Morphol., 31(1):17783, 2013a.

Ramírez, L. M. \& Ballesteros, L. E. Anthropometry of the incus and incudomalleolar and incudostapedial joints in humans: A direct anatomical study. Int. J. Morphol., 31(2):539-45, 2013 b.

Rodríguez Vásquez, J. F.; Merida Velasco, J. R. \& Jiménez Collado, J. Relationships between the temporomandibular joint and the middle ear in human fetuses. J. Dent. Res., 72(1):62-6, 1993.

Sarrat, R.; Torres, A.; Guzman, A. G.; Lostalé, F. \& Whyte, J. Functional structure of human auditory ossicles. Acta Anat. (Basel.), 144(3):189-95, 1992.

Singh, K.; Chhabra, S.; Sirohiwal, B. L. \& Yadav, S. P. S. Morphometry of malleus a possible tool in sex determination. $J$. Forensic Res., 3:152, 2012.

Unur, E.; Ülger, H. \& Ekinci, N. Morphometrical and morphological variations of middle ear ossicles in the newborn. Erciyes Med. J., 24(2):57-63, 2002.

Vinayachandra, P. H.; Viveka, S.; Sudha, M. J.; Balakrishna, S.; Santhosh, K. \& Srinivasa, S. Morphometry and variations of malleus with clinical correlations. Int. J. Anat. Res., 2(1):191-4, 2014.

Whyte, J.; Cisneros, A.; Yus, C.; Obón, J.; Whyte, A.; Serrano, P.; Pérez-Castejón, C. \& Vera, A. Development of the dynamic structure (force lines) of the middle ear ossicles in human foetuses. Histol. Histopathol., 23(9):1049-60, 2008.

\section{Dirección para Correspondencia: \\ Dr. Octavio Binvignat G. \\ Talca \\ CHILE}

Email: obinvignatg@uautonoma.cl

Recibido : 16-02-2015

Aceptado: 28-05-2015 